University of Nebraska - Lincoln

DigitalCommons@University of Nebraska - Lincoln

May 2002

\title{
Magnetoresistance study in thin zig zag NiFe wires
}

J. L. Tsai

Academia Sinica, Nankang 115 Taipei, Taiwan

S.F. Lee

Academia Sinica, Nankang 115 Taipei, Taiwan

Y.D. Yao

Academia Sinica, Nankang 115 Taipei, Taiwan

C. Yu

Academia Sinica, Nankang 115 Taipei, Taiwan

Sy_Hwang Liou

University of Nebraska-Lincoln, sliou@unl.edu

Follow this and additional works at: https://digitalcommons.unl.edu/physicsliou

Part of the Physics Commons

Tsai, J. L.; Lee, S.F.; Yao, Y.D.; Yu, C.; and Liou, Sy_Hwang, "Magnetoresistance study in thin zig zag NiFe wires" (2002). Si-Hwang Liou Publications. 76.

https://digitalcommons.unl.edu/physicsliou/76

This Article is brought to you for free and open access by the Research Papers in Physics and Astronomy at DigitalCommons@University of Nebraska - Lincoln. It has been accepted for inclusion in Si-Hwang Liou Publications by an authorized administrator of DigitalCommons@University of Nebraska - Lincoln. 


\title{
Magnetoresistance study in thin zig zag NiFe wires
}

\author{
J. L. Tsai, ${ }^{a)}$ S. F. Lee, Y. D. Yao, and C. Yu \\ Institute of Physics, Academia Sinica, Nankang 115 Taipei, Taiwan
}

S. H. Liou

Department of Physics, University of Nebraska, Lincoln, Nebraska 68588

Thickness dependence on the domain wall resistivity of zig zag thin permalloy wires was studied from 10 to $300 \mathrm{~K}$. The maximum domain wall resistivity was obtained in wire with $100-\mathrm{nm}$-film thickness. The multidomain state resistivity was $14.29 \mu \Omega \mathrm{cm}$, while single-domain state resistivity was $14.36 \mu \Omega \mathrm{cm}$ at $10 \mathrm{~K}$. The ratio of domain wall magnetoresistance was measured to be $0.034 \%$, $0.112 \%$, and $0.258 \%$, and the magnetic field where the domain wall started to switch was measured as $-70,-40$, and +80 Oe for wires with thicknesses of 20,40 , and $100 \mathrm{~nm}$, respectively, at 250 K. Domain wall resistivity was nearly independent of temperature for wire with 40-nm-film thickness but varied significantly with temperature for 100 -nm-thick wire between 10 and $300 \mathrm{~K}$.

(C) 2002 American Institute of Physics. [DOI: 10.1063/1.1453318]

\section{INTRODUCTION}

The development of lithography techniques makes it possible to fabricate well-defined nanometer-size dots, chains, and wires. It provides a good way to explore the interplay between electron transport and magnetic properties in mesoscopic magnetic devices. The contribution of domain walls to magnetoresistance (MR) has been studied at low temperature in $\mathrm{Ni}$ nanowires. ${ }^{1}$ Resistivity due to domain walls was pointed out to be associated with macroscopic quantum tunneling. ${ }^{2}$ Negative domain wall MR was reported in Fe and Co nanowires. ${ }^{3,4}$ In theory, the negative MR effect was proposed within the linear response theory, where decoherence of weakly localized electrons play an important role. ${ }^{5}$ In particular, magnetic domain structure could be well controlled in zig zag Co and $\mathrm{Ni}_{80} \mathrm{Fe}_{20}$ nanowire by applied magnetic field with different angle orientation in the plane of films. ${ }^{6-8}$ Discontinuous jumps in the wire resistivity were observed and proved as the nucleation and movement of domain walls from multidomain to single-domain state.

In this article, we utilize zig zag $\mathrm{Ni}_{80} \mathrm{Fe}_{20}$ wires with different film thicknesses to explore the temperature dependence of the domain wall contribution to MR. In addition, the thickness dependence of the switching fields and domain wall MR jumps at these switching fields is discussed.

\section{EXPERIMENT}

Field emission electron-beam lithography (Hitachi 4200) and lift-off process as used to prepare $\mathrm{Ni}_{80} \mathrm{Fe}_{20}$ zig zag wires on $\mathrm{Si}(100)$ with $100 \mathrm{~nm} \mathrm{SiO}_{2}$ buffer layers. PMMA and copolymer were spin coated onto the substrate under a proper speed and baked at $135^{\circ} \mathrm{C}$. After the e-beam writing process methyl isobutyl keyton: IPA isopropylalcohol $(\mathrm{MIBK})=1: 3$ and IPA as used to develop the patterns. $\mathrm{Ni}_{80} \mathrm{Fe}_{20}$ films were deposited by thermal evaporation with a base pressure of $1 \times 10^{-6}$ Torr.

\footnotetext{
a) Author to whom correspondence should be addressed; electronic mail: tsaijl@phys.sinica.edu.tw
}

Wires with a pitch of $4 \mu \mathrm{m}$ were zig zagged between two gold pads separated by $50 \mu \mathrm{m}$. Samples with the same line-width of $500 \mathrm{~nm}$ and different thicknesses of 20, 40, 100 , and $150 \mathrm{~nm}$ were fabricated. The total length of these wires are nearly the same, $L=73 \pm 1 \mu \mathrm{m}$. Therefore, the number of corners for each wire is 25 .

MR measurements were made by four-point methods in a variable temperature and magnetic field platform (PPMS, Quantum Design Model 6000) in the temperature range 10$300 \mathrm{~K}$. The magnetic field was applied in the plane and the field angle is measured in the transverse direction of the wires. Resistance at $20 \mathrm{kOe}$ was used as the reference when calculating MR percentage, and detailed investigation was performed from -1500 to 1500 Oe. At room temperature, magnetic domain observation was made by magnetic force microscopy (MFM, NT-MDT Slolver P47) and the field was applied in the longitudinal or transverse directions before measurement.

\section{RESULTS AND DISCUSSION}

The wire consists of 26 straight segments and the length between the neighboring corners, i.e., the pitch, is $4 \mu \mathrm{m}$. From the MFM image in Fig. 1, alternating black and white areas were observed at the corners of the zig zag wire in its remanent state after a magnetic field was applied in the transverse direction of the wire. This indicates that a multidomain magnetic structure is induced by the shape anisotropy of each segment. Figure 2 shows the normalized MR curves of

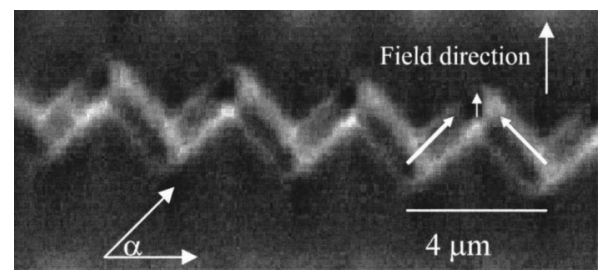

FIG. 1. The magnetic force microscopy image of a $\mathrm{Ni}_{80} \mathrm{Fe}_{20}$ zig zag wire which consists of 25 corners and with a thickness of $40 \mathrm{~nm}$. 

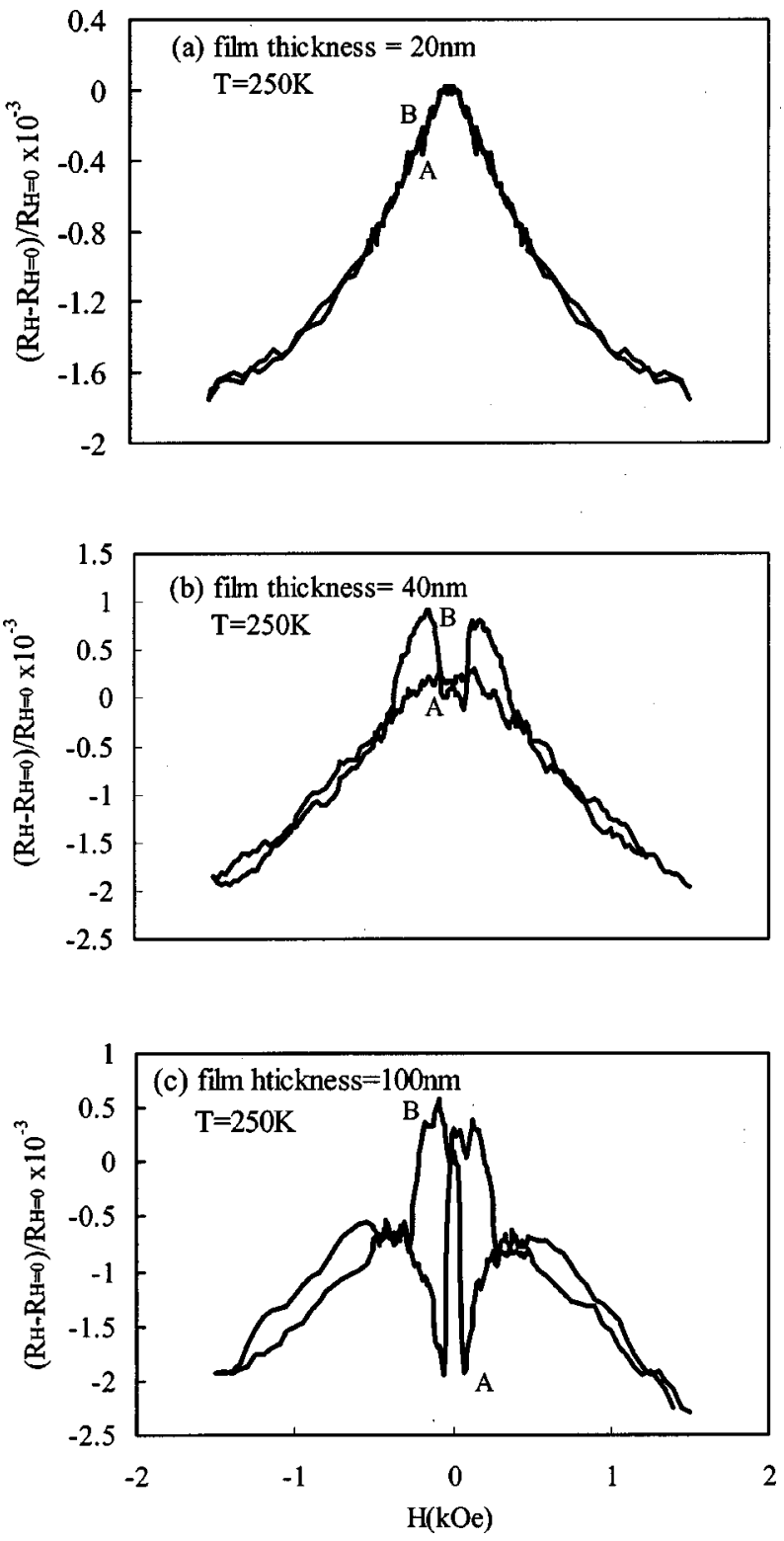

FIG. 2. The normalized MR curves of $\mathrm{Ni}_{80} \mathrm{Fe}_{20}$ zig zag wires at $250 \mathrm{~K}$ with different film thicknesses (a) $20 \mathrm{~nm}$, (b) $40 \mathrm{~nm}$, and (c) $100 \mathrm{~nm}$.

the $\mathrm{Ni}_{80} \mathrm{Fe}_{20}$ zig zag wires at $250 \mathrm{~K}$ with different film thicknesses. The field was applied in the transverse orientation of the wires with the maximum field $20 \mathrm{kOe}$ to eliminate any magnetic history effect. The wires nearly saturate at $3 \mathrm{kOe}$, therefore, the change of the normalized MR in an applied magnetic field between 3 and 20 kOe was very small. As the field is reduced from $1.5 \mathrm{kOe}$ and then reversed, there is a resistivity minimum (a dip) around $+80 \sim-70$ Oe as shown in Figs. 2(a), 2(b), and 2(c). The multidomain-type configuration at corners (point A in Fig. 2) is related to the dips as pointed out by Taniyama et al. ${ }^{6,7}$ As the reversed field is increased, domain wall switches and the MR jumps to a single-domain-type state (point B) in Fig. 2) that corresponds to the maximum resistivity. The ratio of domain wall MR is measured to be $0.034 \%, 0.112 \%$, and $0.258 \%$ in zig zag wires with thickness of 20,40 , and $100 \mathrm{~nm}$. The magnetic

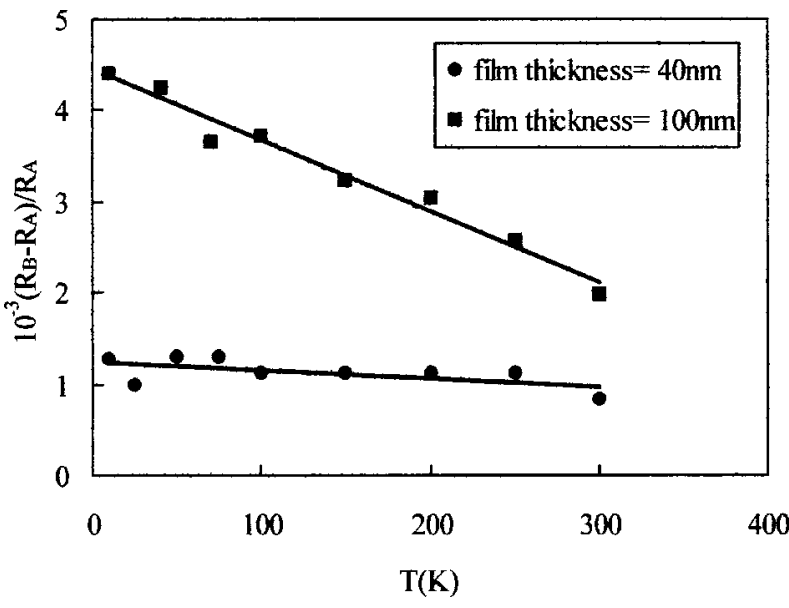

FIG. 3. Temperature dependence of the domain wall MR in zig zag wire with different thicknesses.

field at which the domain wall initiates to switch was measured as $-70,-40$, and +80 Oe for wires with thickness of 20,40 , and $100 \mathrm{~nm}$, respectively.

Figure 3 shows the temperature dependence on the MR ratio from the multidomain state to the single-domain state. The influence of temperature on domain wall MR with different film thickness was substantially different. In Fig. 3, the wire with $100 \mathrm{~nm}$ film thickness has larger $d R / R$ in the temperature range from $10-300 \mathrm{~K}$. This phenomenon can be elucidated by the difference of domain wall type for different film thickness. The Bloch and Néel domain wall width as a function of permalloy film thickness can be calculated by the equation ${ }^{9}$ as follows:

$$
\begin{aligned}
& \gamma_{\text {Block }}=\frac{1}{2} \gamma_{0}\left(\frac{\delta}{\delta_{0}}+\frac{\delta_{0}}{\delta}\right)+\frac{2 \pi \delta^{2} M_{s}^{2}}{\delta+t}, \\
& \gamma_{\text {Náel }}=\frac{1}{2} \gamma_{0}\left(\frac{\delta}{\delta_{0}}+\frac{\delta_{0}}{\delta}\right)+\frac{2 \pi t \delta M_{s}^{2}}{\delta+t},
\end{aligned}
$$

where $\gamma_{0}$ and $\delta_{0}$ are the bulk wall energy and bulk wall width with the value of $0.1 \mathrm{erg} / \mathrm{cm}^{2}$ and $2 \mu \mathrm{m}$, respectively. $M_{s}$ is the magnetization of permalloy film. $\delta$ and $t$ are the domain wall width and thickness of films. By minimizing the total energy, which takes into account the magnetostatic energy, exchange energy, and anisotropy energy, domain wall width with different film thickness is estimated and listed in Table I. According to the $\mathrm{Ni}_{80} \mathrm{Fe}_{20}$ domain wall phase diagram, different types of domain walls dominate at different $\mathrm{Ni}_{80} \mathrm{Fe}_{20}$ film thicknesses. For very thick permalloy films, the domain wall is dominated by Bloch-type in the film center and terminated by Néel caps at the film surface. With reducing film thickness, the domain wall turns into an asymmetric Bloch wall (vortex wall), then into a complex cross-tie wall, and finally for very thin films, into a symmetric Néel wall. For zig zag wires with 100 -nm-film thickness, asymmetric Bloch wall dominates and the calculated wall width is 50 $\mathrm{nm}$. The wall width $(50 \mathrm{~nm})$ is smaller than the wire width $(500 \mathrm{~nm})$ and the film thickness $(100 \mathrm{~nm})$. As a result, the domain wall density is high at the corners. Therefore, domain wall scattering is significant in this film. In contrast, for 
TABLE I. The characteristic length scale of $\mathrm{Ni}_{80} \mathrm{Fe}_{20}$ zig zag wires at $250 \mathrm{~K}$.

\begin{tabular}{ccccc}
\hline \hline Film thickness & Bloch wall (Ref. 9) & Néel wall (Ref. 9) & Mean free path & $10^{-3}\left(R_{B}-R_{A}\right) / R_{A}$ \\
\hline $20 \mathrm{~nm}$ & $10 \mathrm{~nm}$ & $17000 \mathrm{~nm}$ & $<0.2 \mathrm{~nm}$ & 0.34 \\
$40 \mathrm{~nm}$ & $20 \mathrm{~nm}$ & $9000 \mathrm{~nm}$ & $0.85 \mathrm{~nm}$ & 1.12 \\
$100 \mathrm{~nm}$ & $50 \mathrm{~nm}$ & $40 \mathrm{~nm}$ & $20.5 \mathrm{~nm}$ & 2.58 \\
Bulk & $2000 \mathrm{~nm}$ & $\cdots$ & $2.57 \mathrm{~nm}$ & 20 \\
\hline \hline
\end{tabular}

wires with 40 or $20 \mathrm{~nm}$ film thickness, complex cross-tie wall or symmetric Néel wall should dominate and the wall width should be large. Domain wall scattering is not substantial because of low domain wall density. Our MFM currently does not have good enough resolution nor can it work at high magnetic field. Detailed domain structure analysis under field will be carried out in the future.

Our bulk $\mathrm{Ni}_{80} \mathrm{Fe}_{20}$ (thick, unpatterned film) has an estimated mean free path of $\sim 2.57 \mathrm{~nm}$ according to the free electron model with the parameters of free sphere radius, Bohr radius, and resistivity. ${ }^{10}$ Estimated mean free paths of the zig zag wires are also listed in Table I. The values of mean free path are much smaller than the domain sizes and domain wall width. For the wire with 100-nm-film thickness, the mean free path is about $2.05 \mathrm{~nm}$. The slope of MR versus temperature curve in Fig. 3 is related to the domain wall width that is correlated to the intrinsic properties of materials such as saturation moment $\left(M_{S}\right)$, anisotropic constant $(K)$, and exchange stiffness constant $(A)$. The wire with $100-n m-$ film thickness has a much larger slope than that with 40-nmfilm thickness $\left[\delta\left(R_{B}-R_{A}\right) / R_{A} / \delta T=7.8 \times 10^{-3}>0.9\right.$ $\left.\times 10^{-3}\right]$. It implies that the domain wall MR is more sensitive to the variation of temperature in the wire with $100 \mathrm{~nm}$ film thickness. As a result, effective anisotropy of zig zag wires is a function a film thickness.

Static domain wall MR was also proved in this experiment by measuring the angular dependence of resistance at remnant state. High resistivity was observed at the angle range from $0^{\circ}-45^{\circ}\left(\alpha=1^{\circ}-45^{\circ}\right.$ in Fig. 1), and the resistivity was slightly lower from $45^{\circ}-75^{\circ}$ that reflected the single domain state. In contrast, as the field was applied in the orientation of $75^{\circ}-90^{\circ}$, much lower resistivity was obtained. This evidenced static domain wall MR by changing the angle between the applied field and current.

In summary, domain wall resistivity as a function of film thickness was studied by zig zag wire, which can control the domain configuration well, in the article. Different domain wall types dominated in different film thicknesses that significantly influenced the domain wall resistivity.

${ }^{1}$ K. Hong and N. Giordano, J. Magn. Magn. Mater. 15, 1396 (1995).

${ }^{2}$ K. Hong and N. Giordano, Phys. Rev. B 51, 9855 (1995).

${ }^{3}$ U. Ruediger, J. Yu, and A. D. Kent, Appl. Phys. Lett. 73, 1298 (1998).

${ }^{4}$ U. Ruediger, J. Yu, S. Zhang, and A. D. Kent, Phys. Rev. Lett. 80, 5639 (1998).

${ }^{5}$ G. Tatara and H. Fukuyama, Phys. Rev. Lett. 78, 3773 (1997).

${ }^{6}$ T. Taniyama, I. Nakatani, T. Yakabe, and Y. Yamazaki, Appl. Phys. Lett. 76, 613 (2000).

${ }^{7}$ T. Taniyama, I. Nakatani, T. Yakabe, and Y. Yamazaki, Phys. Rev. Lett. 82, 2780 (1999)

${ }^{8}$ J. L. Tsai, S. F. Lee, Y. D. Yao, and C. Yu, J. Magn. Magn. Mater. (in press).

${ }^{9}$ R. L. Comstock, Introduction to Magnetism and Magnetic Recording (Wiley, New York, 1999), pp. 205-206.

${ }^{10}$ N. W. Ashcroft and N. D. Mermin, Solid State Physics (Harcourt College, New York, 1976), p. 757. 